\title{
New Polymorphs of ROY and New Record for Coexisting Polymorphs of Solved Structures
}

\author{
Shuang Chen, Ilia A. Guzei, and Lian Yu* \\ University of Wisconsin - Madison, School of Pharmacy, 777 Highland Ave, Madison WI \\ $53705-2222$ \\ *To whom correspondence should be addressed
}

\section{Supporting Information}

Crystallographic data for ROY polymorph YT04 .......................................................... S2

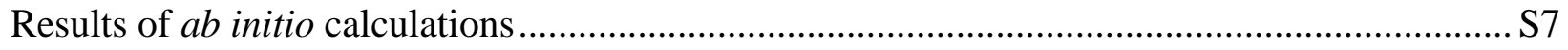

Cambridge Structural Database search for crystal polymorphs............................................... S8 
Figure S1. ORTEP representation of Form YT04 with atomic thermal ellipsoids drawn at the $50 \%$ possibility level.

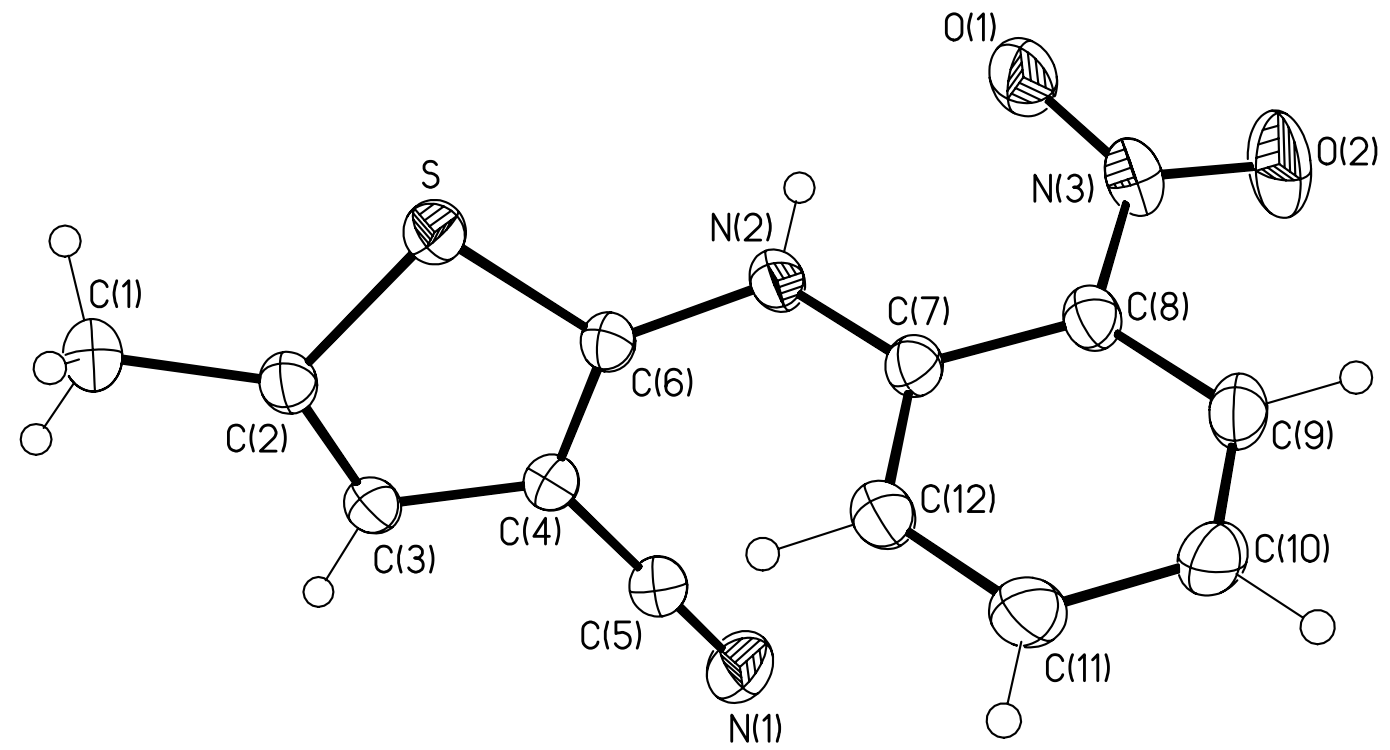


Table S1. Crystal data and structure refinement for Form YT04.

\begin{tabular}{|c|c|c|}
\hline Empirical formula & $\mathrm{C}_{12} \mathrm{H}_{9} \mathrm{~N}_{3} \mathrm{O}_{2} \mathrm{~S}$ & \\
\hline Formula weight & 259.28 & \\
\hline Temperature & 296(2) K & \\
\hline Wavelength & $0.71073 \AA$ & \\
\hline Crystal system & Monoclinic & \\
\hline Space group & $\mathrm{P} 2_{1} / \mathrm{n}$ & \\
\hline Unit cell dimensions & $\begin{array}{l}\mathrm{a}=8.2324(4) \AA \\
\mathrm{b}=11.8173(5) \AA \\
\mathrm{c}=12.3121(6) \AA\end{array}$ & $\begin{array}{l}\alpha=90^{\circ} . \\
\beta=102.5050(10)^{\circ} \\
\gamma=90^{\circ} .\end{array}$ \\
\hline $\begin{array}{l}\text { Volume } \\
\text { Z }\end{array}$ & $\begin{array}{l}1169.36(9) \AA^{3} \\
4\end{array}$ & \\
\hline Density (calculated) & $1.473 \mathrm{Mg} / \mathrm{m}^{3}$ & \\
\hline $\begin{array}{l}\text { Absorption coefficient } \\
F(000)\end{array}$ & $\begin{array}{l}0.273 \mathrm{~mm}^{-1} \\
536\end{array}$ & \\
\hline Crystal size & $0.45 \times 0.31 \times 0.26$ & \\
\hline Theta range for data collection & 2.42 to $26.36^{\circ}$. & \\
\hline Index ranges & $-9<=\mathrm{h}<=10,-9<=\mathrm{h}$ & $-7<=\mathrm{l}<=15$ \\
\hline Reflections collected & 5068 & \\
\hline Independent reflections & $2312[\mathrm{R}(\mathrm{int})=0.0$ & \\
\hline Completeness to theta $=26.36^{\circ}$ & $96.7 \%$ & \\
\hline Absorption correction & Multi-scan with S & \\
\hline Max. and min. transmission & 0.9323 and 0.8868 & \\
\hline $\begin{array}{l}\text { Refinement method } \\
\text { Data / restraints / parameters }\end{array}$ & $\begin{array}{l}\text { Full-matrix least-s } \\
2312 / 0 / 164\end{array}$ & on $\mathrm{F}^{2}$ \\
\hline Goodness-of-fit on $\mathrm{F}^{2}$ & 1.069 & \\
\hline Final $\mathrm{R}$ indices $[\mathrm{I}>2 \operatorname{sigma}(\mathrm{I})]$ & $\mathrm{R} 1=0.0387, \mathrm{wR} 2$ & \\
\hline $\mathrm{R}$ indices (all data) & $\mathrm{R} 1=0.0430, \mathrm{wR} 2$ & \\
\hline Largest diff. peak and hole & 0.238 and $-0.319 e$ & \\
\hline
\end{tabular}


Table S2. Atomic coordinates ( $\left.\mathrm{x} 10^{4}\right)$ and equivalent isotropic displacement parameters $\left(\AA^{2} \mathrm{x}\right.$ $10^{3}$ ) for Form YT04. U(eq) is defined as one third of the trace of the orthogonalized $\mathrm{U}^{\mathrm{ij}}$ tensor.

\begin{tabular}{lccrl}
\hline & $\mathrm{x}$ & $\mathrm{y}$ & $\mathrm{z}$ & $\mathrm{U}(\mathrm{eq})$ \\
\hline $\mathrm{S}$ & $4589(1)$ & $7742(1)$ & $352(1)$ & $44(1)$ \\
$\mathrm{O}(1)$ & $5818(2)$ & $8799(1)$ & $4326(1)$ & $72(1)$ \\
$\mathrm{O}(2)$ & $5631(2)$ & $8146(2)$ & $5910(1)$ & $83(1)$ \\
$\mathrm{N}(1)$ & $8255(2)$ & $4917(2)$ & $2828(1)$ & $68(1)$ \\
$\mathrm{N}(2)$ & $5349(2)$ & $7430(1)$ & $2596(1)$ & $46(1)$ \\
$\mathrm{N}(3)$ & $5383(2)$ & $8059(1)$ & $4899(1)$ & $51(1)$ \\
$\mathrm{C}(1)$ & $5188(2)$ & $7054(2)$ & $-1698(1)$ & $52(1)$ \\
$\mathrm{C}(2)$ & $5486(2)$ & $6858(1)$ & $-467(1)$ & $40(1)$ \\
$\mathrm{C}(3)$ & $6467(2)$ & $6077(1)$ & $157(1)$ & $40(1)$ \\
$\mathrm{C}(4)$ & $6530(2)$ & $6196(1)$ & $1320(1)$ & $39(1)$ \\
$\mathrm{C}(5)$ & $7493(2)$ & $5491(1)$ & $2158(1)$ & $48(1)$ \\
$\mathrm{C}(6)$ & $5568(2)$ & $7073(1)$ & $1555(1)$ & $39(1)$ \\
$\mathrm{C}(7)$ & $4541(2)$ & $6793(1)$ & $3256(1)$ & $38(1)$ \\
$\mathrm{C}(8)$ & $4547(2)$ & $7068(1)$ & $4373(1)$ & $40(1)$ \\
$\mathrm{C}(9)$ & $3712(2)$ & $6398(2)$ & $5011(1)$ & $48(1)$ \\
$\mathrm{C}(10)$ & $2851(2)$ & $5467(2)$ & $4570(2)$ & $54(1)$ \\
$\mathrm{C}(11)$ & $2825(2)$ & $5178(2)$ & $3472(2)$ & $54(1)$ \\
$\mathrm{C}(12)$ & $3656(2)$ & $5818(1)$ & $2835(1)$ & $46(1)$ \\
& & & & \\
\hline
\end{tabular}


Table S3. Anisotropic displacement parameters $\left(\AA^{2} \times 10^{3}\right)$ for Form YT04. The anisotropic displacement factor exponent takes the form: $-2 \pi^{2}\left[\mathrm{~h}^{2} \mathrm{a}^{* 2} \mathrm{U}^{11}+\ldots+2 \mathrm{~h} \mathrm{k} \mathrm{a}^{*} \mathrm{~b} * \mathrm{U}^{12}\right]$

\begin{tabular}{lcccccc}
\hline & $\mathrm{U} 11$ & $\mathrm{U}^{22}$ & $\mathrm{U}^{33}$ & $\mathrm{U}^{23}$ & $\mathrm{U} 13$ & $\mathrm{U} 12$ \\
\hline $\mathrm{S}$ & $50(1)$ & $43(1)$ & $41(1)$ & $2(1)$ & $15(1)$ & $7(1)$ \\
$\mathrm{O}(1)$ & $107(1)$ & $57(1)$ & $58(1)$ & $-17(1)$ & $32(1)$ & $-27(1)$ \\
$\mathrm{O}(2)$ & $94(1)$ & $118(1)$ & $39(1)$ & $-24(1)$ & $16(1)$ & $-32(1)$ \\
$\mathrm{N}(1)$ & $86(1)$ & $63(1)$ & $53(1)$ & $15(1)$ & $13(1)$ & $18(1)$ \\
$\mathrm{N}(2)$ & $65(1)$ & $39(1)$ & $40(1)$ & $-7(1)$ & $25(1)$ & $-9(1)$ \\
$\mathrm{N}(3)$ & $50(1)$ & $64(1)$ & $40(1)$ & $-11(1)$ & $15(1)$ & $-5(1)$ \\
$\mathrm{C}(1)$ & $65(1)$ & $54(1)$ & $36(1)$ & $-1(1)$ & $11(1)$ & $-1(1)$ \\
$\mathrm{C}(2)$ & $47(1)$ & $39(1)$ & $36(1)$ & $-3(1)$ & $13(1)$ & $-7(1)$ \\
$\mathrm{C}(3)$ & $49(1)$ & $35(1)$ & $38(1)$ & $-3(1)$ & $16(1)$ & $-2(1)$ \\
$\mathrm{C}(4)$ & $47(1)$ & $35(1)$ & $36(1)$ & $1(1)$ & $13(1)$ & $-2(1)$ \\
$\mathrm{C}(5)$ & $61(1)$ & $43(1)$ & $42(1)$ & $1(1)$ & $17(1)$ & $3(1)$ \\
$\mathrm{C}(6)$ & $47(1)$ & $37(1)$ & $35(1)$ & $-1(1)$ & $15(1)$ & $-4(1)$ \\
$\mathrm{C}(7)$ & $39(1)$ & $39(1)$ & $36(1)$ & $2(1)$ & $13(1)$ & $4(1)$ \\
$\mathrm{C}(8)$ & $40(1)$ & $45(1)$ & $36(1)$ & $-1(1)$ & $11(1)$ & $6(1)$ \\
$\mathrm{C}(9)$ & $52(1)$ & $57(1)$ & $39(1)$ & $7(1)$ & $19(1)$ & $11(1)$ \\
$\mathrm{C}(10)$ & $61(1)$ & $49(1)$ & $62(1)$ & $8(1)$ & $33(1)$ & $1(1)$ \\
$\mathrm{C}(11)$ & $59(1)$ & $44(1)$ & $64(1)$ & $-5(1)$ & $27(1)$ & $-8(1)$ \\
$\mathrm{C}(12)$ & $54(1)$ & $44(1)$ & $44(1)$ & $-6(1)$ & $18(1)$ & $-3(1)$ \\
& & & & & & \\
\hline
\end{tabular}


Table S4. Hydrogen coordinates ( $\left.x 10^{4}\right)$ and isotropic displacement parameters $\left(\AA^{2} \times 10^{3}\right)$ for Form YT04.

\begin{tabular}{lrrrr}
\hline & $\mathrm{x}$ & $\mathrm{y}$ & $\mathrm{z}$ & $\mathrm{U}(\mathrm{eq})$ \\
\hline $\mathrm{H}(2)$ & 5738 & 8081 & 2837 & 55 \\
$\mathrm{H}(1 \mathrm{~A})$ & 5885 & 6558 & -2014 & 77 \\
$\mathrm{H}(1 \mathrm{~B})$ & 5445 & 7826 & -1838 & 77 \\
$\mathrm{H}(1 \mathrm{C})$ & 4042 & 6904 & -2031 & 77 \\
$\mathrm{H}(3)$ & 7041 & 5519 & -139 & 48 \\
$\mathrm{H}(9)$ & 3747 & 6591 & 5748 & 57 \\
$\mathrm{H}(10)$ & 2287 & 5029 & 4995 & 65 \\
$\mathrm{H}(11)$ & 2236 & 4542 & 3163 & 64 \\
$\mathrm{H}(12)$ & 3631 & 5599 & 2106 & 55 \\
\hline
\end{tabular}


Table S5. Ab initio Conformational Energies and Dipole Moments of ROY ${ }^{\mathrm{a}}$

\begin{tabular}{|c|c|c|c|c|c|c|}
\hline Reference & $\theta_{\text {thio }}$ & $\theta_{\text {phen }}$ & $\theta_{\text {nitro }}$ & $E-E_{\min }, \mathrm{kJ} / \mathrm{mole}$ & $\mu, \mathrm{D}$ & $E-E_{Y}, \mathrm{~kJ} / \mathrm{mole}$ \\
\hline \multirow[t]{8}{*}{ Barrier 1} & 0.0 & -130.1 & -23.8 & 7.29 & 8.06 & \\
\hline & 10.0 & -136.1 & -22.4 & 5.82 & 7.94 & \\
\hline & 21.7 & -143.5 & -20.5 & 4.36 & 7.80 & \\
\hline & 35.0 & -149.1 & -17.3 & 3.07 & 7.61 & \\
\hline & 46.1 & -155.0 & -13.9 & 2.14 & 7.50 & \\
\hline & 52.6 & -156.4 & -12.8 & 1.66 & 7.34 & \\
\hline & 60.0 & -159.6 & -10.1 & 1.12 & 7.28 & \\
\hline & 75.0 & -166.9 & -2.5 & 0.28 & 7.18 & \\
\hline \multirow[t]{7}{*}{ Minimum } & 88.4 & -174.6 & 0.7 & 0.00 & 7.05 & \\
\hline & 104.4 & 176.2 & 4.5 & 0.36 & 6.77 & \\
\hline & 112.8 & 173.2 & 7.4 & 0.77 & 6.55 & \\
\hline & 120.0 & 168.1 & 9.4 & 1.30 & 6.35 & \\
\hline & 135.0 & 160.1 & 13.5 & 2.99 & 5.77 & \\
\hline & 150.0 & 148.8 & 17.2 & 5.86 & 5.12 & \\
\hline & 165.0 & 137.1 & 20.4 & 10.06 & 4.36 & \\
\hline Barrier 2 & 180.0 & 123.4 & 24.2 & 15.28 & 3.54 & \\
\hline$\overline{\mathrm{R}}$ & 21.7 & -150.0 & -18.4 & 4.69 & 8.14 & 3.85 \\
\hline ORP & 39.4 & -174.3 & -3.5 & 6.43 & 8.15 & 5.60 \\
\hline OP & 46.1 & -167.3 & -18.7 & 3.33 & 7.91 & 2.49 \\
\hline ON & 52.6 & -173.3 & -4.4 & 3.48 & 8.00 & 2.64 \\
\hline YN & 104.1 & -175.2 & -3.6 & 0.87 & 6.69 & 0.03 \\
\hline $\mathrm{Y}$ & 104.7 & -175.0 & -1.8 & 0.84 & 6.68 & 0.00 \\
\hline YT04 & 112.8 & -169.7 & -14.7 & 1.00 & 6.63 & 0.16 \\
\hline
\end{tabular}

${ }^{a}$ Torsional angles in bold are fixed during geometry optimization. Calculations were performed at the RHF/6-31G* level using Spartan ’02. 


\section{Cambridge Structural Database Search for Polymorphic Systems with More Than Three Members}

We searched the Cambridge Structural Database (Feb. 2005) ${ }^{1}$ for polymorphic systems with three or more members. We began by retrieving all entries containing the qualifier "polymorph", "form", "phase”, or "modification". We included structures of one or more components determined at room or other temperatures, but excluded organometallic compounds and entries for which atomic coordinates are unavailable. After sorting the resulting 4717 hits by hand, we identified 118 compounds with three or more polymorphs (see list below). Of these, 102 are trimorphic, 14 are tetramorphic $(*)$, one is pentamorphic (SUTHAZ), and one is hexamorphic (QAXMEH).

$\begin{array}{lllll}\text { ADULEQ* } & \text { DIMETH } & \text { HADKIG } & \text { MEZKEH } & \text { SULAMD } \\ \text { AMBACO* } & \text { DIWWEL } & \text { HEYHU** } & \text { MNIAAN } & \text { SUTHAZ (n=5) } \\ \text { AMNTPY } & \text { DIYJUQ } & \text { HIMWIJ } & \text { NADQAL } & \text { TEPHTH } \\ \text { AWAKIS } & \text { DLABUT } & \text { HYQUIN } & \text { NAGHOT } & \text { THIOUR } \\ \text { AZADAG } & \text { DLMSUC } & \text { IFULUQ* } & \text { NAPYMA } & \text { TNBENZ } \\ \text { BANHOO } & \text { DMANTL } & \text { IJETOG } & \text { NIMFOE } & \text { TORSEM } \\ \text { BEWKUJ* } & \text { DMFUSC } & \text { IMDIAC } & \text { NOJHEZ } & \text { TPEPHO } \\ \text { BIMYAX } & \text { DMMTCN } & \text { IVADUE } & \text { OCHTET } & \text { TUHBAZ } \\ \text { BISMEV } & \text { DOBTUJ } & \text { JATFUF } & \text { PARQUI } & \text { TURPYB } \\ \text { BIXGIY* } & \text { DPYRAM } & \text { JIBCIG } & \text { PCBZAM } & \text { UDAYUT } \\ \text { BOPKOG } & \text { DUCKOB } & \text { JUSBUU } & \text { PHTHCY } & \text { UJORIU } \\ \text { BZCHOL } & \text { DUVFUV } & \text { KAXHAS* } & \text { PNEOSI } & \text { UNEWUF } \\ \text { CBMZPN* } & \text { DUVZOJ } & \text { KTCYQM } & \text { PUBMUU } & \text { VISKAJ* } \\ \text { CENRIW } & \text { ESTRON } & \text { LAURAC } & \text { PUPBAD } & \text { XINBEB } \\ \text { CILHIO* } & \text { FACRIK } & \text { LAVMOK } & \text { PYRZIN* } & \text { YERRUI } \\ \text { COMXAD } & \text { FAFWIS } & \text { LILXIN } & \text { QAXMEH (n=6) } & \text { YUYHIJ } \\ \text { DATREV } & \text { FAWFOY } & \text { MABZNA* } & \text { QOGNEF } & \text { ZEPFAB } \\ \text { DBEZLM } & \text { FEGWAP } & \text { MACCID } & \text { RBTCNQ } & \text { ZEXREZ } \\ \text { DCBFRO } & \text { FESKAP } & \text { MALEHY } & \text { RUWYIR* } & \text { ZOGQAN } \\ \text { DCLANT } & \text { FIDYIA } & \text { MBPHOL } & \text { SAMPYM } & \text { ZZZHWI } \\ \text { DCLBEN } & \text { FILGEM } & \text { MBYINO } & \text { SIFLOI } & \text { ZZZIYE } \\ \text { DEGGEB } & \text { GADSIO } & \text { MBZYAN } & \text { SIKLIH } & \text { ZZZVTY } \\ \text { DETBAA } & \text { GISRIJ } & \text { MCHTEP } & \text { SOBPEE } & \\ \text { DHNAPH } & \text { GLYCIN } & \text { METHOL } & \text { STARAC* } & \end{array}$

The pentamorphic system of 1:2 guest-host inclusion compound 1,3-dichloropropane/tris (5-acetyl-3-thienyl) methane (TATM) ${ }^{2}$ has not been deposited in the CSD. The current CSD contains three polymorphs of carbamazepine, but a fourth was reported in $2003 .^{3}$

\footnotetext{
${ }^{1}$ Allen, F. H. Acta Cryst. 2002, B58, 380-388. The latest Full Release (November 2004, CSD V5.26) has 325,709 entries. The February 2005 Intermediate Release has 12,736 entries. The total number of entries is now 338,445.

${ }^{2}$ Sidhu, P. S.; Enright, G. D.; Udachin, K. A.; Ripmeester, J. A. Crystal Growth \& Design 2004, 4, 1249-1257.

${ }^{3}$ Grzesiak, A. L.; Lang, M.; Kim, K.; Matzger, A. J. J. Pharm. Sci. 2003, 92, 2260-2271.
} 